\title{
(1)
}

\section{Caution necessary when prescribing fibrates in the elderly}

A population-based cohort study suggests that fibrate use is associated with increases in serum creatinine level among elderly patients. The investigators suggest that caution should therefore be exercised when prescribing fibrates to these patients, by starting the prescription at a low dosage and closely monitoring renal function.

Fibrates are commonly used to treat dyslipidemia and can reduce the risk of major cardiovascular events. However, case reports, small clinical studies and randomized, controlled trials indicate that fibrate use is associated with an increase in serum creatinine level. "Elderly patients have a high prevalence of dyslipidemia and chronic kidney disease (CKD) and are frequently excluded from randomized trials", write the authors of this study. The investigators therefore carried out this study, published in Annals of Internal Medicine, to characterize acute renal outcomes associated with fibrate use in elderly patients and the effect of these outcomes on health-care use. "Our primary objective was to examine the association between a new prescription for fibrate and renal outcomes within 90 days."

The investigators used four databases to provide information about elderly residents of Ontario, Canada with new prescriptions for a fibrate or ezetimibe. Ezetimibe was chosen as the comparator drug because it is also considered a second-line therapy after a statin for treatment of dyslipidemia.

Patients aged $\geq 66$ years with at least one prescription for either a fibrate or ezetimibe between 1 January 2004 and 31 December 2008 were identified. In total, 80,903 individuals met the inclusion criteria $(19,072$ receiving a first prescription for a fibrate and 61,831 for ezetimibe). All patients were followed for 90 days. The primary outcome was hospitalization for an increase in serum creatinine level. Secondary outcomes were consultation with a nephrologist, receipt of dialysis for severe acute kidney injury

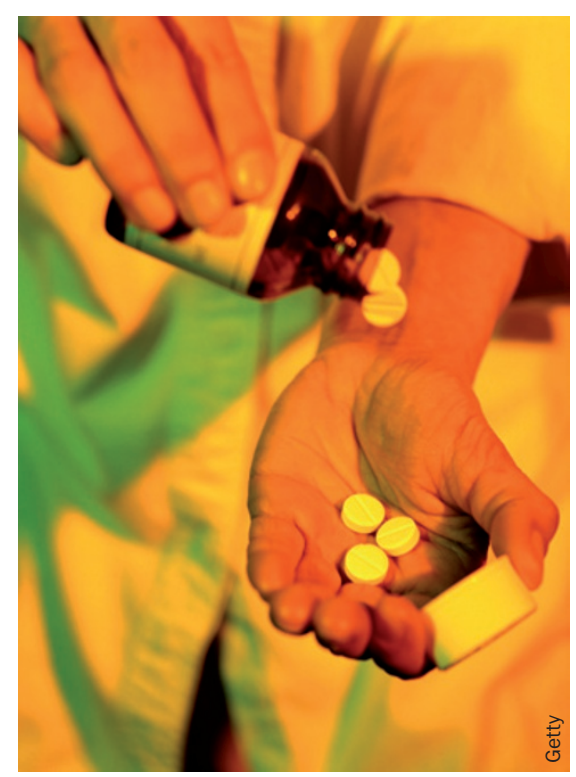

(AKI), all-cause mortality and an increase in serum creatinine level of $\geq 50 \%$ (in those patients who had serum creatinine levels measured before and within 90 days of a new fibrate or ezetimibe prescription).

Fibrate users had a higher risk of hospitalization for an increase in serum creatinine level within 90 days of a new prescription than did ezetimibe users (adjusted odds ratio 2.4). Patients receiving fibrates were also more likely to consult a nephrologist than were those receiving ezetimibe (adjusted odds ratio 1.3). No difference was found between the groups in terms of receiving dialysis for severe AKI or all-cause mortality.

Among patients with at least one measurement of serum creatinine before and after prescription of the new drug, $9.1 \%$ of fibrate users and $0.3 \%$ of ezetimibe users had an increase in serum creatinine levels of $\geq 50 \%$. This difference translates into an almost 30 -fold increased risk of $\mathrm{a} \geq 50 \%$ increase in serum creatinine concentration in the fibrate group. The presence of diabetes mellitus or statin use did not influence the association between fibrate use and hospitalization for an increase in serum creatinine level. By contrast, patients with CKD were more likely to require hospitalization for an increase in serum creatinine level after fibrate use than were those without CKD. Similarly, patients with CKD were significantly more likely to consult a nephrologist than were those without CKD. Patients receiving a fibrate with a baseline estimated glomerular filtration rate $(\mathrm{eGFR})<60 \mathrm{ml} / \mathrm{min} / 1.73 \mathrm{~m}^{2}$ had greater absolute increases in serum creatinine level than did those with an $\mathrm{eGFR} \geq 60 \mathrm{ml} / \mathrm{min} / 1.73 \mathrm{~m}^{2}$.

The authors also carried out a systematic search of other studies that reported renal outcomes related to fibrate exposure, including the ACCORD, FIELD and LEADER trials. Compared with the studies identified in their search, the patients in Zhao et al.'s cohort were older, had a higher prevalence of CKD, were more likely to be receiving a higher dose of fibrate given their renal function, and were less likely to have their renal function monitored at regular intervals.

Limitations of this study included its observational design, the differences between fibrates and ezetimibe that preclude a direct comparison, and the fact that most patients received fenofibrate, which means that the results might not apply to other fibrates.

However, the researchers believe that their results provide drug safety data in elderly patients with CKD.

"The mechanism for the increase in serum creatinine level remains poorly understood", write Zhao and colleagues. "Until we have a better understanding of the underlying mechanism by which fibrates increase serum creatinine levels and its long-term renal effects, we believe that, when fibrates are prescribed to older patients, it would be prudent to start the prescription at a low dosage and arrange for close monitoring of renal function, as has been done in clinical trials."

Helene Myrvang

Original article Zhao, Y. Y. et al. New fibrate use and acute renal outcomes in elderly adults: a population-based study. Ann. Intern. Med. 156, 560-569 (2012) 